\title{
On the imaginary simple roots of the Borcherds algebra $\mathfrak{g}_{I_{9,1}}$
}

\author{
Oliver Bärwald ${ }^{\mathrm{a}, 1}$, Reinhold W. Gebert ${ }^{\mathrm{b}, 2}$, Hermann Nicolai ${ }^{\mathrm{c}, 3}$ \\ a Department of Mathematics, King's College London, Strand, London WC2R 2LS, Great Britain \\ ${ }^{\mathrm{h}}$ Institute for Advanced Study, School of Natural Sciences, Olden Lane, Princeton, NJ 08540, USA \\ c Max-Planck-Institut für Gravitationsphysik, Albert-Einstein-Institut, Schlaatzweg 1, D-14473 Potsdam, \\ Germany
}

Received 2 June 1997; revised 12 September 1997; accepted 12 September 1997

\begin{abstract}
In a recent paper [O. Bärwald, R.W. Gebert, M. Günaydin and H. Nicolai, preprint KCL-MTH97-22, IASSNS-HEP-97/20, PSU-TH-178, AEI-029, hep-th/9703084, to appear in Commun. Math. Phys.] it was conjectured that the imaginary simple roots of the Borcherds algebra $\mathrm{g}_{I_{9,1}}$ at level 1 are its only ones. We here propose an independent test of this conjecture, establishing its validity for all roots of norm $\geqslant-8$. However, the conjecture fails for roots of norm -10 and beyond, as we show by computing the simple multiplicities down to norm -24 , which turn out to be remarkably small in comparison with the corresponding $E_{10}$ multiplicities. Our derivation is based on a modified denominator formula combining the denominator formulas for $E_{10}$ and $\mathfrak{g}_{I_{9,1}}$, and provides an efficient method for determining the imaginary simple roots. In addition, we compute the $E_{10}$ multiplicities of all roots up to height 231 , including levels up to $\ell=6$ and norms -42. (C) 1998 Elsevier Science B.V.
\end{abstract}

PACS: $11.25 . \mathrm{H} ; 02.10 . \mathrm{S}$

Keywords: Hyperbolic Kac-Moody algebras; String vertex operators

\section{Introduction}

In this paper we begin a systematic study of the simple imaginary roots of the Borcherds Lie algebra $\mathfrak{g}_{I_{9,1}}$ and propose a new method to compute their (simple)

\footnotetext{
${ }^{1}$ Supported by Gottlieb Daimler- und Karl Benz-Stiftung under Contract No. 02-22/96.

${ }^{2}$ Supported by Deutsche Forschungsgemeinschaft under Contract No. DFG Ge 963/1-1.

${ }^{3}$ Supported in part by EU Contract FMRX-CT96-0012.
} 
multiplicities, enabling us to test the conjecture made in Ref. [1] concerning the set of imaginary simple roots of $\mathfrak{g}_{I_{9,1}}$. Let us recall that $\mathfrak{g}_{I_{9,1}}$ is the Lie algebra of physical states of a subcritical bosonic string fully compactified on the even self-dual Lorentzian lattice $I I_{9,1}$, which coincides with the root lattice $Q\left(E_{10}\right)$ of the hyperbolic Kac-Moody algebra $E_{10}$; this lattice is spanned by the simple roots $\boldsymbol{r}_{-1}, \boldsymbol{r}_{0}, \boldsymbol{r}_{1}, \ldots, \boldsymbol{r}_{8}$ of $E_{10}$ (alias the real simple roots of $\left.\mathfrak{g}_{H_{9,1}}\right)$. Our primary motivation for investigating the root system of $\mathfrak{g}_{l_{9,1}}$ is to better understand the hyperbolic algebra $E_{10}$, which is the maximal Kac-Moody algebra contained in $\mathfrak{g}_{l_{9,1}}$ :

$$
E_{10} \subset \mathfrak{g}_{I_{9,1}} .
$$

As explained in Ref. [1], the difficulties of understanding hyperbolic Kac-Moody algebras on the one hand and Borcherds algebras (also called generalized Kac-Moody algebras [3]) on the other hand are to some extent complementary: while a Lorentzian Kac-Moody algebra has a well-understood root system, but the structure of the algebra and its root spaces (including their dimensions!) is very complicated, Borcherds algebras may possess a simple realization in terms of physical string states, but usually have a very complicated root system due to the appearance of imaginary (i.e. non-positive norm) simple roots. The Chevalley generators corresponding to imaginary simple roots of $\mathfrak{g}_{I_{9,1}}$ are needed to complete the subalgebra $E_{10}$ to the full Lie algebra of physical states. This can be seen by decomposing the vector space

$$
\mathcal{M}:=\mathfrak{g}_{l_{9,1}} \ominus E_{10},
$$

into an infinite direct sum of "missing modules" all of which are highest or lowest weight modules with respect to the subalgebra $E_{10}$ (see Refs. [1,11]). This implies that all of $\mathfrak{g}_{I I_{9,1}}$ can be generated from the highest and lowest weight states by the action of (i.e. multiple commutation with) the $E_{10}$ raising or lowering operators. However, not all the lowest weight states in $\mathcal{M}$ correspond to imaginary simple roots of $\mathfrak{g}_{I_{9,1}}$. This is because the commutation of two lowest weight states yields another lowest weight state; yet it is only those lowest weight states which cannot be obtained as multiple commutators of previous states and which must therefore be added "by hand" that will give rise to new imaginary simple roots. Complete knowledge of the imaginary simple roots of $\mathfrak{g}_{H_{0,1}}$ is thus tantamount to understanding the hyperbolic Kac-Moody algebra $E_{10}$ (or at least its root multiplicities).

Let us pause for a moment to rephrase these statements in string theory language. As has been shown in Ref. [9], commutation of two physical string states in these completely compactified string models is equivalent to tree-level scattering. So, starting with a set of ten fundamental tachyons (associated with the real simple roots), we generate by multiple scattering an infinite set of physical string states at arbitrary mass level. By construction, this set is just the hyperbolic algebra $E_{10}$, and it is easy to see that it contains all tachyonic and all massless states as these can be produced by elementary scattering processes. By contrast, $E_{10}$ does not exhaust the massive states because not all of these can be obtained by scattering $E_{10}$ states of lower mass. To be sure, $E_{10}$ does 
act via the adjoint action on all physical states, i.e. we can scatter two states only one of which belongs to $E_{10}$ to get another state, which is also not in $E_{10}$. Therefore, the remaining ("missing", or "decoupled") part of the spectrum can be decomposed into $E_{10}$ representations. In order to identify the pertinent highest or lowest weight states, the strategy is to pick suitable missing string states of lowest mass and add them as extra Lie algebra elements to $E_{10}$. Since the momenta of these states have negative norm $^{4}$ this corresponds to adjoining timelike simple roots to $E_{10}$. These simple roots generically come with multiplicities bigger than one because massive string states have additional polarization degrees of freedom, whereas the tachyons are scalars, and the real simple roots consequently have multiplicity one always. Following Ref. [1] we will designate the simple multiplicity of an imaginary simple root $\boldsymbol{\Lambda}$ by $\mu(\boldsymbol{\Lambda})$; this simple multiplicity $\mu(\boldsymbol{A})$ must not be confused with the multiplicity mult $(\boldsymbol{\Lambda})$ of $\boldsymbol{\Lambda}$ as a root of $E_{10}$ or with the multiplicity $\operatorname{dim} \mathfrak{g}_{H_{\mathrm{g}, 1}}{ }^{(\boldsymbol{\Lambda})}$ of $\boldsymbol{\Lambda}$ as a root of $\mathfrak{g}_{H_{\mathrm{g}, 1}}$. Therefore, the Lie algebra of all physical states is no longer a Kac-Moody algebra since the Cartan matrix may now have negative integers on the diagonal. In general, the above procedure has to be repeated an infinite number of times because by scattering the adjoined massive states with $E_{10}$ states, we still do not exhaust the whole spectrum.

So far, there are only a few string models for which the root system of the associated Borcherds algebra has been completely analyzed, and for which a complete set of imaginary simple roots associated with extra string states, including their multiplicities, has been identified. Celebrated examples are the fake monster [4] and the monster Lie algebra [5], which are related to a toroidal and an orbifold compactification of the 26-dimensional bosonic string. In Ref. [1], an infinite set of level-1 imaginary simple roots (with exponentially growing, known multiplicities) for the Borcherds algebra $\mathfrak{g}_{H_{y, 1}}$ was found and it was conjectured that this set should exhaust all of them. The results of this paper disprove the conjecture and show that the structure of the $\mathfrak{g}_{/ t_{9,1}}$ root system is more involved than originally thought. In establishing these results, we are led to explore the multiplicities and simple multiplicities of these algebras much further than has been done before. Our calculations of the simple root multiplicities are based on a new denominator formula which combines the known denominator formulas for $E_{10}$ and $\mathfrak{g}_{l_{1,1}}$. Although not as efficient as the Peterson recursion formula (which appears to have no analog for simple multiplicities), this formula does simplify the computations substantially and allows us to evaluate the simple multiplicities down to norms -24 . Since we have made no attempt to optimize our computer program (using the symbolic algebra system Maple V) with regard to speed, it is quite conceivable that the calculations can be carried even further. As an important by-product of this investigation we have determined the $E_{10}$ root multiplicities up to height 231 including levels up to $\ell=6$ and norms down to -42 , because these numbers are needed as an input in our modified denominator formula. Since these results may also be of use in other contexts, we have tabulated them separately in Appendix A.

\footnotetext{
${ }^{4}$ By the "norm" of a root $\boldsymbol{A}$ we mean the (Minkowskian) scalar product $\boldsymbol{A}^{2}$.
} 
Although the ultimate pattern underlying the multiplicities remains elusive, and despite the failure of our original conjecture, our results do exhibit some intriguing features. In particular, we would like to draw attention to the fact that, as far as we have computed them, the simple multiplicities come out to be remarkably small, both in comparison with the $E_{10}$ multiplicities and with the number of decoupled states. For instance, at level $\ell=4$, we find

$$
\mu\left(\boldsymbol{\Lambda}_{3}\right)=2,
$$

whereas the $E_{10}$ multiplicity of $\boldsymbol{A}_{3}$ is given by

$$
\operatorname{mult}\left(\boldsymbol{\Lambda}_{3}\right)=1044218
$$

and the number of associated decoupled states is equal to

$$
\Delta\left(\boldsymbol{\Lambda}_{3}\right)=278125
$$

This behavior is to be contrasted with the gnome Lie algebra $\mathfrak{g}_{I_{1,1}}$ for which the simple root multiplicities and the root space dimensions are of the same order of magnitude [1]. On the other hand, the pattern is clearly more irregular than for the simple imaginary roots of the fake monster algebra $\mathfrak{g}_{I_{25,1}}$ whose simple multiplicities either vanish, or are uniformly equal to 24 . In fact, at this point, we cannot even exclude the possibility of a "chaotic" behavior at yet larger (negative) norms and higher levels - after all, $E_{10}$ is defined by means of a recursive procedure just like simple chaotic systems. Being optimistic and barring such pathologies, the supreme challenge is now to unveil the secret behind the numbers we have found; and, whatever hypothesis is proffered to explain $E_{10}$, it must be tested against these numbers.

\section{A modified denominator formula}

We first summarize our notations and conventions for $E_{10}$, mostly following [13] to which we refer the reader for further details. The real simple roots $\boldsymbol{r}_{i}$ and the fundamental weights $\boldsymbol{\Lambda}_{i}$ are labeled in accordance with the Coxeter-Dynkin diagram:

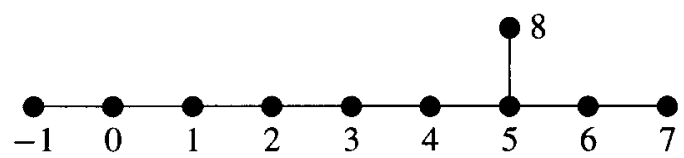

from which the $E_{10}$ Cartan matrix $A_{i j}$ can be easily read off. The root $r_{-1}$ which extends the affine subalgebra $E_{9}$ to the full hyperbolic algebra $E_{10}$ will be referred to as the over-extended root. The level $\ell$ of a root $r$ in the fundamental Weyl chamber is defined by

$$
\ell:=-\delta \cdot \boldsymbol{r},
$$


where $\delta$ denotes the affine null root. The fundamental Weyl chamber $\mathcal{C}$ is the positive convex cone in $I_{9,1}$ generated by the fundamental weights $\boldsymbol{\Lambda}_{i}=-\sum_{j}\left(A^{-1}\right)_{i j} \boldsymbol{r}_{j}>0$, obeying $\boldsymbol{\Lambda}_{i} \cdot \boldsymbol{r}_{j}=-\boldsymbol{\delta}_{i j}$ (this is the only place where we deviate from the conventions of Ref. [13]). So we have, for instance, $\boldsymbol{\Lambda}_{-1}=\boldsymbol{\delta}$ and $\boldsymbol{\Lambda}_{0}=\boldsymbol{r}_{-1}+2 \boldsymbol{\delta}$, etc.; since $\boldsymbol{\Lambda}_{-1}^{2}=0$ and $\boldsymbol{\Lambda}_{i}^{2}<0$ for $i \geqslant 0, \mathcal{C}$ lies inside the forward lightcone and touches it at one edge. Acting on $\mathcal{C}$ with all elements of the $E_{10}$ Weyl group and taking the closure of the resulting set, one obtains the so-called Tits cone which coincides with the full forward light-cone containing all imaginary roots [12]. The Weyl chamber $\mathcal{C}$ contains in particular the imaginary simple roots which must be adjoined to complete $E_{10}$ to the full algebra $\mathfrak{g}_{l_{9,1}}$ of physical states. For the determination of root multiplicities it is therefore sufficient to restrict attention to roots in the fundamental Weyl chamber $\mathcal{C}$; for a given root norm the computation can thus be reduced to a a finite number of checks.

In Ref. [1] a complete characterization of all level-1 imaginary simple roots of $\mathfrak{g}_{H_{9,1}}$ and their multiplicities was given: the missing lowest weight states are just the purely longitudinal physical states with momenta $\boldsymbol{r}_{-1}+N \boldsymbol{\delta}$ for $N \geqslant 2$. The multiplicities of these simple roots are given by $\mu\left(\boldsymbol{r}_{-1}+N \boldsymbol{\delta}\right)=\pi_{1}(N)$, where

$$
\pi_{d}(n):=p_{d}(n)-p_{d}(n-1),
$$

with

$$
\sum_{n=0}^{\infty} p_{d}(n) q^{n}=\prod_{n \geqslant 1}\left(1-q^{n}\right)^{-d} .
$$

One would expect the structure to be far more involved at higher levels, but the explicit calculations in Ref. [1] revealed that there were no imaginary simple roots $s$ at level 2 with $s^{2} \geqslant-6$. This unexpected result prompted the conjecture that the level-1 roots of $\mathfrak{g}_{I_{\mathrm{l}, \mathrm{l}}}$ are in fact the only imaginary simple roots of $\mathfrak{g}_{I_{\mathfrak{g}, 1}}$, or, equivalently, that the set of missing lowest weight states is the free Lie algebra generated by the purely longitudinal states at level 1. The evidence presented in Ref. [1] was based on computer calculations of commutators of certain level-1 states but this method becomes impractical beyond the examples studied there because of the rapidly increasing algebraic complexity as the root norms become more negative. We here present an independent test via a modified denominator formula, enabling us to carry the checks much further, even without use of a computer. This new formula combines the $E_{10}$ denominator formula with the one for $\mathfrak{g}_{/ I_{y, 1}}$, and is therefore sensitive only to the "difference" of these two algebras.

The denominator formula for $E_{10}$ reads (see e.g. Ref. [12])

$$
\prod_{\boldsymbol{r} \in \Delta_{t}}\left(1-\mathrm{e}^{r}\right)^{\operatorname{mult}(\boldsymbol{r})}=\sum_{\mathfrak{w} \in \mathfrak{W}}(-1)^{\mathfrak{w}} \mathrm{e}^{\mathfrak{w}(\boldsymbol{\rho})-\boldsymbol{\rho}},
$$

where $\Delta_{+}$are the positive roots of $E_{10}$ and $\boldsymbol{\rho}$ is the $E_{10}$ Weyl vector, i.e. $\boldsymbol{\rho} \cdot \boldsymbol{r}_{i}=-\frac{1}{2} \boldsymbol{r}_{i}^{2}$ for $i=-1,0,1, \ldots, 8$. The determination of the root multiplicities

$$
\operatorname{mult}(\boldsymbol{r}):=\operatorname{dim} E_{10}^{(r)}
$$


at arbitrary level $\ell$ remains an unsolved problem for $E_{10}$ (and, more generally, for any hyperbolic Kac-Moody algebra): closed formulas exist only for levels $|\ell| \leqslant 2$ [13], and, albeit in implicit form, for $\ell= \pm 3$ [2]. The denominator formula relates the infinite product over all positive roots to an infinite sum over the Weyl group $\mathfrak{W} \equiv \mathfrak{W}\left(E_{10}\right)$ generated by the reflections with respect to the real simple roots. In principle, all root multiplicities can be determined from it by multiplying out the 1.h.s. and comparing the resulting expressions term by term, but in practice this method reaches its limits rather quickly. However, one can derive from (6) the so-called Peterson recursion formula (see e.g. Ref. [14]), which can be implemented on a computer. Because, to the best of our knowledge, explicit tables of $E_{10}$ multiplicities available in the literature stop at $|\ell|=2[13]$ and the actual numbers are needed in our calculation, we have computed the $E_{10}$ multiplicities of all roots up to height 231 and levels $\leqslant 6$ by putting the Peterson formula on a computer. Readers may notice that, beyond $\ell=2$, these multiplicities are no longer functions of the norm alone, as was still the case for the level 2 roots (so Murphy's law has struck again).

For the Borcherds algebra $\mathfrak{g}_{H_{9,1}}$, the denominator formula must be amended in two ways: firstly, the $E_{10}$ multiplicities $\operatorname{mult}(\boldsymbol{r})$ are replaced by the corresponding numbers of physical states

$$
\operatorname{dim} \mathfrak{g}_{H_{9,1}}{ }^{(\boldsymbol{r})}=\pi_{9}\left(1-\frac{1}{2} \boldsymbol{r}^{2}\right) \geqslant \operatorname{mult}(\boldsymbol{r}),
$$

and secondly, the r.h.s. must be supplemented by extra terms due to the imaginary simple roots. The modified denominator formula reads [3]

$$
\prod_{r \in \Delta_{+}}\left(1-\mathrm{e}^{r}\right)^{\pi_{9}\left(1-\frac{1}{2} r^{2}\right)}=\sum_{\mathfrak{w} \in \mathfrak{W}}(-1)^{\mathfrak{w}} \mathrm{e}^{\mathfrak{w}(\rho)-\rho} \sum_{s} \epsilon(s) \mathrm{e}^{\mathfrak{w}(s)},
$$

where $\epsilon(s)$ is $(-1)^{n}$ if $s$ is a sum of $n$ distinct pairwise orthogonal imaginary simple roots of $\mathfrak{g}_{H_{9,1}}$, and zero otherwise. As already pointed out the candidates for imaginary simple roots are all lattice points in the fundamental Weyl chamber $\mathcal{C}$. Because all massless physical string states belong to $E_{10}$, there are no lightlike simple roots. Consequently, the imaginary simple roots are all timelike, and we therefore conclude that there are no pairwise orthogonal imaginary simple roots. Hence

$$
\begin{aligned}
\sum_{s} \epsilon(s) \mathrm{e}^{\mathfrak{w}(s)} & =1-\sum_{\substack{\boldsymbol{i} \in \mathcal{C} \cap u_{g, 1} \\
\boldsymbol{\Lambda}^{2} \leqslant-2}} \mu(\boldsymbol{\Lambda}) \mathrm{e}^{\mathfrak{w}(\boldsymbol{A})} \\
& =1-\sum_{N=2}^{\infty} \pi_{1}(N) \mathrm{e}^{\mathfrak{w}(\boldsymbol{r}-1+N \delta)}+\ldots,
\end{aligned}
$$

where in the second line of this equation, we have made use of our knowledge of the simple roots at level 1 and their multiplicities; the dots stand for possible contributions from higher-level imaginary simple roots. Unfortunately, there seems to be no analog of the Peterson recursion formula that would allow a (comparatively) quick determination of the imaginary roots and their simple multiplicities. The proof of this formula crucially 
relies upon the existence of a Weyl vector with $\boldsymbol{\rho} \cdot \boldsymbol{s}=-\frac{1}{2} s^{2}$ for all simple roots $s$ : while this requirement is met by the ten simple roots of $E_{10}$ it fails already for the level-1 imaginary simple roots $\boldsymbol{s}=\boldsymbol{r}_{-1}+N \boldsymbol{\delta}$, as one can easily check. Consequently, no Weyl vector exists for $\mathfrak{g}_{H_{9,1}}$ and we have to seek another way of simplifying the denominator formula in order to test our conjecture.

The idea is to modify the formula in such a way that it "measures" only the corrections that arise when enlarging $E_{10}$ to the full Lie algebra $\mathfrak{g}_{l_{l, 1}}$ of physical states. To this aim let us introduce the difference between the $\mathfrak{g}_{I_{9,1}}$ and the $E_{10}$ multiplicities, i.e., the number of decoupled (missing) states associated with the root $r$,

$$
\Delta(\boldsymbol{r}):=\pi_{9}\left(1-\frac{1}{2} \boldsymbol{r}^{2}\right)-\operatorname{mult}(\boldsymbol{r}) \quad(\geqslant 0) .
$$

So, using the known results in for $|\ell| \leqslant 2$, we have

$$
\Delta(\boldsymbol{r})= \begin{cases}0 & \text { for } \ell=0, \\ \pi_{9}\left(1-\frac{1}{2} \boldsymbol{r}^{2}\right)-p_{8}\left(1-\frac{1}{2} \boldsymbol{r}^{2}\right) & \text { for } \ell=1, \\ \pi_{9}\left(1-\frac{1}{2} r^{2}\right)-\xi\left(3-\frac{1}{2} r^{2}\right) & \text { for } \ell=2,\end{cases}
$$

where the function $\xi(n)$ was defined and tabulated in [13] (notice the accidental equality $\left.p_{8}(3)=\xi(5)\right)$. The explicit $E_{10}$ multiplicities beyond level 2 which we need have been collected in the table of Appendix A, where we also list the relevant values for $\Delta(r)$.

Inserting the $E_{10}$ denominator formula into the one for $\mathfrak{g}_{\mu_{9,1}}$, we obtain the following formula after a little algebra:

$$
\begin{aligned}
& \left(\sum_{\mathfrak{w} \in \mathfrak{W}}(-1)^{\mathfrak{w}} \mathrm{e}^{\mathfrak{w}(\boldsymbol{\rho})}\right)\left(\prod_{r \in \Delta_{+}}\left(1-\mathrm{e}^{r}\right)^{\Delta(\boldsymbol{r})}-1\right) \\
& =-\sum_{\mathfrak{w} \in \mathfrak{W}} \sum_{\substack{\boldsymbol{A} \in \mathcal{C} \cap u_{\mathfrak{g}, 1} \\
\boldsymbol{\Lambda}^{2} \leqslant-2}}(-1)^{\mathfrak{T}} \mu(\boldsymbol{\Lambda}) \mathrm{e}^{\mathfrak{m}(\boldsymbol{p}+\boldsymbol{\Lambda})} \\
& =-\sum_{\mathfrak{w} \in \mathfrak{W}} \sum_{N=0}^{\infty}(-1)^{\mathfrak{w}} \pi_{1}(N+2) \mathrm{e}^{\mathfrak{m}\left(\boldsymbol{\rho}+\boldsymbol{\Lambda}_{0}+N \boldsymbol{A}_{-1}\right)}+\ldots
\end{aligned}
$$

The dots stand for the level $\ell \geqslant 2$ contributions which were conjectured to vanish in Ref. [1]. We will show explicitly how the conjecture fails by exhibiting non-zero contributions of this type.

One advantage of this formula is the absence of terms without $\boldsymbol{r}_{-1}$ on the 1.h.s. since for all such roots we have $\Delta(\boldsymbol{r})=0$; thus, the $E_{9}$ part of the denominator formula has already been factored out in (13). Given the $E_{10}$ root multiplicities it allows us to determine the simple roots together with their simple multiplicities rather efficiently, as we will demonstrate in the following section. The analysis of (13) can be considerably simplified by restricting oneself on the r.h.s. to roots in the fundamental Weyl chamber $\mathcal{C}$. To see this, we note that in terms of the fundamental weights we have $\boldsymbol{\rho}=\sum_{j=-1}^{8} \boldsymbol{\Lambda}_{j}$, 
so that $\boldsymbol{\rho}+\boldsymbol{\Lambda} \in \mathcal{C}$ for all $\boldsymbol{\Lambda} \in \mathcal{C}$, and that for any $\mathfrak{w} \neq 1$ the vector $\mathfrak{w}(\boldsymbol{\rho}+\boldsymbol{\Lambda})$ lies outside the fundamental Weyl chamber since no fundamental reflection leaves $\rho$ invariant. With this observation, the sum over the Weyl group on the r.h.s. can be disregarded.

The general procedure for evaluating the new denominator formula is then as follows. Let us fix a dominant integral level- $\ell$ weight $\boldsymbol{\Lambda} \in \mathcal{C}$ for which $\boldsymbol{\Lambda}=\sum m_{j} \boldsymbol{\Lambda}_{j}$ with $m_{j} \geqslant 0$. We wish to determine the coefficient of $\mathrm{e}^{\Lambda}$ on the 1.h.s. of formula (13). To do so, we must first look for all possible decompositions $\boldsymbol{\rho}+\boldsymbol{\Lambda}=\mathfrak{w}(\boldsymbol{\rho})+\boldsymbol{v}$ with $\boldsymbol{v} \in Q_{+}\left(E_{10}\right)$. The reason why we cannot drop the sum over the Weyl group on the l.h.s., even if we consider only terms in the fundamental Weyl chamber on the r.h.s., is that in this decomposition neither $\mathfrak{w}(\boldsymbol{\rho})$ nor $v$ will in general be in the fundamental Weyl chamber even if their sum is. Now, for

$$
\mathfrak{w}(\boldsymbol{\rho})=\boldsymbol{\rho}+\boldsymbol{a}
$$

we have $a>0$ unless $\mathfrak{w}=1$; this follows from the fact that the Weyl vector is a dominant $E_{10}$ weight. From the preservation of the scalar product and basic properties of the Weyl vector we deduce that (for $\boldsymbol{a} \neq \mathbf{0}$ )

$$
a^{2}=-2 \boldsymbol{\rho} \cdot \boldsymbol{a}=2 \mathrm{ht}(\boldsymbol{a})>0,
$$

where "ht" denotes the height of the root, and

$$
v^{2}=\boldsymbol{\Lambda}^{2}+2(\text { ht }(\boldsymbol{a})-\boldsymbol{a} \cdot \boldsymbol{\Lambda}) .
$$

Note that for $\boldsymbol{\Lambda} \in \mathcal{C}$ we also have $\boldsymbol{a} \cdot \boldsymbol{\Lambda} \leqslant 0$ for any positive $\boldsymbol{a}$. These simple relations severely constrain the possible $a$ 's that must be taken into account: since repeated Weyl reflections will increase the height, there are only finitely many terms which can contribute for any given $\boldsymbol{A}$. Having found all possible $\boldsymbol{\nu}=\boldsymbol{\Lambda}-\boldsymbol{a} \in Q_{+}\left(E_{10}\right)$ that can appear, the next problem will be to calculate the coefficient of $\mathrm{e}^{v}$ arising from the product over the positive roots. For this we must find all decompositions $v=\sum_{j} v_{j}$ with $v_{j} \in \Delta_{+}$. Some care must be exercised with the various minus signs arising from the Weyl reflections as well as from the binomial expansions of the factors in the product over the positive roots. In particular, we have to know all multiplicities of the relevant positive roots. At higher level, this causes the extra complication of determining in which $E_{10}$ Weyl orbits these roots lie. Given any positive root $r$, this amounts to reflecting it by use of the Weyl group into the fundamental chamber. Although there seem to be no general results available we have found the following method, due to Fuchs [8], to be very efficient. One starts by rewriting the root in the basis of fundamental weights, i.e. $\boldsymbol{r}=\sum_{i} m_{i} \boldsymbol{\Lambda}_{i}$ for $m_{i} \in \mathbb{Z}$. Since $\boldsymbol{r} \notin \mathcal{C}$ by assumption, at least one of the coefficients $m_{i}$ is negative. Choose a negative coefficient with the largest absolute value $m_{k}$, say, and apply the $k$ th fundamental Weyl reflection to the root. We obtain $\mathfrak{w}_{k}(\boldsymbol{r})=\sum_{i} \mathfrak{w}_{k}\left(m_{i}\right) \boldsymbol{A}_{i}$ with $\mathfrak{w}_{k}\left(m_{i}\right):=m_{i}-m_{k} A_{k i}$, so that the coefficient of $\boldsymbol{\Lambda}_{k}$ is now $-m_{k}>0$. The next step is to determine again the most negative coefficient, to apply the corresponding Weyl reflection and so on. This algorithm always terminates after a finite number of steps. 


\section{Sample calculations for $\Lambda^{2} \geqslant-10$}

Let us now illustrate how the calculation works in detail for some simple examples for which the new denominator formula can be evaluated by hand. This is certainly the case for roots $\boldsymbol{A} \in \mathcal{C}$ with $\boldsymbol{\Lambda}^{2} \geqslant-10$, and perhaps beyond; however, the combinatorial complexities, and thus the possible sources of errors, increase rapidly for large negative $\boldsymbol{\Lambda}^{2}$, and we have therefore preferred to let the computer do the rest of the calculation, see the following section.

For the level-1 roots the required computations are quite straightforward, as we need only consider $\mathfrak{w} \in \mathfrak{W}\left(E_{9}\right)$ and make use of the fact that the $E_{9}$ Weyl orbit of $\boldsymbol{r}_{-1}+N \boldsymbol{\delta}$ consists of all elements $\boldsymbol{r}_{-1}+\left(N+\frac{1}{2} \boldsymbol{b}^{2}\right) \boldsymbol{\delta}+\boldsymbol{b}$ with $\boldsymbol{b} \in Q\left(E_{8}\right)$. It is then not difficult to check the validity of (13) for roots $\boldsymbol{A}=\boldsymbol{\Lambda}_{0}+(N-2) \boldsymbol{\Lambda}_{-1}=\boldsymbol{r}_{-1}+N \boldsymbol{\delta}$ to large $N$. However, since we anyhow know the formula to be correct at level 1 , we refrain from giving further details. As regards the level- 2 roots of norm $\geqslant-6$, our calculation will just confirm the conclusions reached in [1], whereas for norms -8 and -10 our results are new (and unlikely to be obtainable by the methods of [1]). We will show that all higher-level terms on the r.h.s. of Eq. (13) down to norm squared -8 are absent, in agreement with the conjecture of [1]; the relevant roots in $\mathcal{C}$ are $\boldsymbol{\Lambda}_{7}, \boldsymbol{\Lambda}_{1}, 2 \boldsymbol{\Lambda}_{0}$ and $\boldsymbol{\Lambda}_{7}+\boldsymbol{\delta}$, all of level 2 . There are two norm -10 roots in $\mathcal{C}$, namely $\boldsymbol{\Lambda}_{1}+\boldsymbol{\delta}$, of level 2, and $\boldsymbol{A}_{8}$, of level 3 (all other level $\geqslant 3$ roots in $\mathcal{C}$ have norm $\leqslant-12$ ); for these, we will find a non-vanishing result, refuting the conjecture of Ref. [1]. Further counterexamples will be provided in the next section.

In the actual calculations we will need to determine to which Weyl orbit a given root belongs, and whenever referring to a root lying in a certain Weyl orbit we have checked this by Fuchs' algorithm. For small norms this is not really necessary, if there is only one Weyl orbit; for instance, there is only one orbit $\mathfrak{W}\left(\boldsymbol{\Lambda}_{0}\right)$, for roots with $r^{2}=-2$ which has $\Delta\left(\boldsymbol{\Lambda}_{0}\right)=1$. For roots with $\boldsymbol{r}^{2}=-4$ there are two Weyl orbits, $\mathfrak{W}\left(\boldsymbol{\Lambda}_{0}+\boldsymbol{\delta}\right)$ and $\mathfrak{W}\left(\boldsymbol{A}_{7}\right)$, which happen to yield the same numbers $\Delta\left(\boldsymbol{\Lambda}_{0}+\delta\right)=\Delta\left(\boldsymbol{\Lambda}_{7}\right)=9$. In fact, all Weyl orbits of level-2 roots in $\mathcal{C}$ with the same norm have the same value for $\Delta$ because the level-2 multiplicities (described by the function $\xi$ ) depend only on the norm. The combinatorial prefactors below arise from the combinatorics of the indices $i, j, \ldots$ and are most conveniently determined by inspection of the Coxeter-Dynkin diagram. Relations such as $0 \leqslant \boldsymbol{a}^{2}<-\boldsymbol{\Lambda}^{2}+2 \boldsymbol{a} \cdot \boldsymbol{A}$ show that we have listed all non-zero contributions to the simple multiplicities of the roots under consideration. Finally, an important consistency check on the calculation is that the non-zero coefficients must come out to be non-positive due to the absence of pairwise orthogonal simple imaginary roots. This applies in particular to the large (negative) norm roots to be analyzed in the next section, where the final result is obtained as an alternating sum of huge contributions.

(1) $\boldsymbol{\Lambda}^{2}=-4$, i.e. $\boldsymbol{A}=\boldsymbol{\Lambda}_{7}$.

(a) $A \cdot a=0$ :

(i) $a=0 \Longrightarrow-\Delta\left(\boldsymbol{A}_{7}\right)=-9$;

(ii) $a^{2}=2$, i.e. $a=r_{i}$ for $i \neq 7 \Longrightarrow 9 \times(-1)^{2} \Delta\left(\Lambda_{0}\right)=9$. 
In total, this gives $-9+9=0$ for the simple multiplicity.

(2) $\boldsymbol{\Lambda}^{2}=-6$, i.e. $\boldsymbol{\Lambda}=\boldsymbol{\Lambda}_{1}$.

(a) $\boldsymbol{\Lambda} \cdot \boldsymbol{a}=0$ :

(i) $a=0 \Longrightarrow-\Delta\left(\Lambda_{1}\right)=-53$;

(ii) $a^{2}=2$, i.e. $a=r_{i}$ for $i \neq 1 \Longrightarrow 9 \times(-1)^{2} \Delta\left(\Lambda_{7}\right)=81$;

(iii) $a^{2}=4$, i.e. $a=r_{i}+r_{j}$ for $i, j \neq 1$ and $r_{i} \cdot r_{j}=0 \Longrightarrow 29 \times(-1)^{3} \Delta\left(A_{0}\right)=$ -29 ;

(b) $\boldsymbol{A} \cdot \boldsymbol{a}=-1$ : (i) $\boldsymbol{a}^{2}=2$, i.e. $a=r_{1} \Rightarrow(-1)^{2} \Delta\left(\boldsymbol{\Lambda}_{0}\right)=1$.

In total, this gives $-53+81-29+1=0$ for the simple multiplicity ${ }^{5}$.

(3) $\boldsymbol{\Lambda}^{2}=-8$, i.e. $\boldsymbol{\Lambda}=2 \boldsymbol{\Lambda}_{0}$ or $\boldsymbol{\Lambda}=\boldsymbol{\Lambda}_{7}+\boldsymbol{\delta}$. Let $\boldsymbol{\Lambda}=2 \boldsymbol{\Lambda}_{0}$.

(a) $\boldsymbol{\Lambda} \cdot \boldsymbol{a}=0$ :

(i) $a=0 \Longrightarrow-\Delta\left(2 \Lambda_{0}\right)=-246$;

(ii) $a^{2}=2$, i.e. $a=r_{i}$ for $i \neq 0 \Longrightarrow(-1)^{2}\left[\Delta\left(\Lambda_{0}+2 \delta\right)+8 \Delta\left(\Lambda_{1}\right)\right]=478$, since $\boldsymbol{\Lambda}-\boldsymbol{r}_{-1} \in \mathfrak{W}\left(\boldsymbol{\Lambda}_{0}+2 \boldsymbol{\delta}\right)$ and $\boldsymbol{\Lambda}-\boldsymbol{r}_{1}, \ldots, \boldsymbol{\Lambda}-\boldsymbol{r}_{8} \in \mathfrak{W}\left(\boldsymbol{\Lambda}_{1}\right)$;

(iii) $a^{2}=4$, i.e. $a=r_{i}+r_{j}$ for $i, j \neq 0$ and $r_{i} \cdot r_{j}=0 \Longrightarrow 29 \times(-1)^{3} \Delta\left(\Lambda_{7}\right)=$ -261 ;

(iv) $\boldsymbol{a}^{2}=6$, i.e. $\boldsymbol{a}=\boldsymbol{r}_{i}+\boldsymbol{r}_{j}+\boldsymbol{r}_{k}$ for $i, j, k \neq 0$ and $\boldsymbol{r}_{i} \cdot \boldsymbol{r}_{j}=\boldsymbol{r}_{j} \cdot \boldsymbol{r}_{k}=\boldsymbol{r}_{k} \cdot \boldsymbol{r}_{i}=0$, or $a=2 \boldsymbol{r}_{i}+\boldsymbol{r}_{j}$ for $i, j \neq 0$ and $\boldsymbol{r}_{i} \cdot \boldsymbol{r}_{j}=-1 \Longrightarrow\left[42 \times(-1)^{4}+14 \times(-1)^{3}\right] \Delta\left(\boldsymbol{A}_{0}\right)=$ 28 ;

(b) $\boldsymbol{\Lambda} \cdot \boldsymbol{a}=-2$ :

$a^{2}=2$, i.e. $a=r_{0} \Longrightarrow(-1)^{2} \Delta\left(\Lambda_{0}\right)=1$.

In total, this gives $-246+478-261+28+1=0$ for the simple multiplicity. Let $\boldsymbol{\Lambda}=\boldsymbol{\Lambda}_{7}+\boldsymbol{\delta}$.

(a) $\boldsymbol{\Lambda} \cdot \boldsymbol{a}=0$ :

(i) $a=0 \Longrightarrow-\Delta\left(A_{7}+\delta\right)=-246$;

(ii) $a^{2}=2$, i.e. $a=r_{i}$ for $i \neq-1,7 \Longrightarrow 8 \times(-1)^{2} \Delta\left(\Lambda_{1}\right)=424$, since $\boldsymbol{\Lambda}-\boldsymbol{a} \in \mathfrak{W}\left(\boldsymbol{A}_{1}\right)$;

(iii) $a^{2}=4$, i.e. $a=r_{i}+r_{j}$ for $i, j \neq-1,7$ and $r_{i} \cdot r_{j}=0 \Rightarrow 21 \times$ $(-1)^{3} \Delta\left(\Lambda_{7}\right)=-189$;

(iv) $\boldsymbol{a}^{2}=6$, i.e. $\boldsymbol{a}=\boldsymbol{r}_{i}+\boldsymbol{r}_{j}+\boldsymbol{r}_{k}$ for $i, j, k \neq-1,7$ and $\boldsymbol{r}_{i} \cdot \boldsymbol{r}_{j}=\boldsymbol{r}_{j} \cdot \boldsymbol{r}_{k}=\boldsymbol{r}_{k} \cdot \boldsymbol{r}_{i}=0$, or $\boldsymbol{a}=2 \boldsymbol{r}_{i}+\boldsymbol{r}_{j}$ for $i, j \neq-1,7$ and $\boldsymbol{r}_{i} \cdot \boldsymbol{r}_{j}=-1 \Longrightarrow\left[21 \times(-1)^{4}+14 \times\right.$ $\left.(-1)^{3}\right] \Delta\left(\Lambda_{0}\right)=7$

(b) $\boldsymbol{A} \cdot \boldsymbol{a}=-1$ :

(i) $a^{2}=2$, i.e. $a=r_{-1}$ or $a=r_{7} \Rightarrow 2 \times(-1)^{2} \Delta\left(\Lambda_{7}\right)=18$;

(ii) $a^{2}=4$, i.e. $a=r_{-1}+r_{i}$ for $i \neq-1,0,7$ or $a=r_{7}+r_{j}$ for $j \neq-1,6,7$ $\Longrightarrow 14 \times(-1)^{3} \Delta\left(\boldsymbol{A}_{0}\right)=-14$.

In total, this gives $-246+424-189+7+18-14=0$ for the simple multiplicity.

(4) $\boldsymbol{\Lambda}^{2}=-10$, i.e. $\boldsymbol{\Lambda}=\Lambda_{1}+\delta$ or $\boldsymbol{A}=\boldsymbol{\Lambda}_{8}$. Let $\boldsymbol{\Lambda}=\boldsymbol{\Lambda}_{1}+\delta$.

(a) $\boldsymbol{\Lambda} \cdot \boldsymbol{a}=0$ :

(i) $a=0 \Longrightarrow-\Delta\left(\boldsymbol{\Lambda}_{1}+\delta\right)+(-1)^{2} \Delta\left(\boldsymbol{\Lambda}_{0}\right) \Delta\left(\boldsymbol{\Lambda}_{0}+r_{0}\right)=-982$,

\footnotetext{
${ }^{5}$ These two examples amply demonstrate the power of formula (13): the necessary commutator calculations in Ref. [1] needed to reach the same conclusion required two hours of CPU time!
} 
since $\boldsymbol{A}=2 \boldsymbol{A}_{0}+\boldsymbol{r}_{0}{ }^{6}$;

(ii) $\boldsymbol{a}^{2}=2$, i.e. $a=r_{i}$ for $i \neq-1,1 \Longrightarrow 8 \times(-1)^{2} \Delta\left(\boldsymbol{A}_{7}+\boldsymbol{\delta}\right)=1968$, since $\boldsymbol{\Lambda}-\boldsymbol{r}_{0} \in \mathfrak{W}\left(2 \boldsymbol{\Lambda}_{0}\right)$ and $\boldsymbol{\Lambda}-\boldsymbol{r}_{i} \in \mathfrak{W}\left(\boldsymbol{\Lambda}_{7}+\boldsymbol{\delta}\right)$ for $i \neq-1,0,1$;

(iii) $\boldsymbol{a}^{2}=4$, i.e. $\boldsymbol{a}=\boldsymbol{r}_{i}+\boldsymbol{r}_{j}$ for $i, j \neq-1,1$ and $\boldsymbol{r}_{i} \cdot \boldsymbol{r}_{j}=0 \Longrightarrow 22 \times$ $(-1)^{3} \Delta\left(\Lambda_{1}\right)=-1166$,

since $\boldsymbol{\Lambda}-\boldsymbol{a} \in \mathfrak{W}\left(\boldsymbol{\Lambda}_{1}\right)$;

(iv) $\boldsymbol{a}^{2}=6$, i.e. $\boldsymbol{a}=\boldsymbol{r}_{i}+\boldsymbol{r}_{j}+\boldsymbol{r}_{k}$ for $i, j, k \neq-1,1$ and $\boldsymbol{r}_{i} \cdot \boldsymbol{r}_{j}=\boldsymbol{r}_{j} \cdot \boldsymbol{r}_{k}=\boldsymbol{r}_{k} \cdot \boldsymbol{r}_{i}=0$, or $\boldsymbol{a}=2 \boldsymbol{r}_{i}+\boldsymbol{r}_{j}$ for $i, j \neq-1,1$ and $\boldsymbol{r}_{i} \cdot \boldsymbol{r}_{j}=-1 \Longrightarrow\left[26 \times(-1)^{4}+12 \times\right.$ $\left.(-1)^{3}\right] \Delta\left(A_{7}\right)=126$;

(v) $a^{2}=8$, i.e. $a=r_{i}+r_{j}+r_{k}+r_{i}$ for $i, j, k, l \neq-1,1$ and $r_{i} \cdot r_{j}=$ $\ldots=\boldsymbol{r}_{l} \cdot \boldsymbol{r}_{i}=0$, or $\boldsymbol{a}=2 \boldsymbol{r}_{i}+\boldsymbol{r}_{j}+\boldsymbol{r}_{k}$ for $i, j, k \neq-1,1$ and $\boldsymbol{r}_{i} \cdot \boldsymbol{r}_{j}=-1$, $\boldsymbol{r}_{i} \cdot \boldsymbol{r}_{k}=\boldsymbol{r}_{j} \cdot \boldsymbol{r}_{k}=0$, or $\boldsymbol{a}=2\left(\boldsymbol{r}_{i}+\boldsymbol{r}_{j}\right)$ for $i, j \neq-1,1$ and $\boldsymbol{r}_{i} \cdot \boldsymbol{r}_{j}=-1$ $\Rightarrow\left[13 \times(-1)^{5}+48 \times(-1)^{4}+6 \times(-1)^{4}\right] \Delta\left(\boldsymbol{A}_{0}\right)=41$;

(b) $\boldsymbol{A} \cdot \boldsymbol{a}=-1$ :

(i) $a^{2}=2$, i.e. $a=r_{-1}$ or $a=r_{1} \Rightarrow(-1)^{2}\left[\Delta\left(\Lambda_{0}+2 \delta\right)+\Delta\left(\Lambda_{1}\right)\right]=107$, since $\boldsymbol{A}-\boldsymbol{r}_{-1} \in \mathfrak{W}\left(\boldsymbol{\Lambda}_{0}+2 \boldsymbol{\delta}\right)$ and $\boldsymbol{\Lambda}-\boldsymbol{r}_{1} \in \mathfrak{W}\left(\boldsymbol{\Lambda}_{\mathfrak{l}}\right)$;

(ii) $a^{2}=4$, i.e. $a=r_{-1}+r_{i}$ for $i \neq-1,0,1$, or $a=r_{1}+r_{j}$ for $j \neq-1,0,1,2$ $\Longrightarrow 13 \times(-1)^{3} \Delta\left(\Lambda_{7}\right)=-117$;

(iii) $a^{2}=6$, i.e. $a=r_{-1}+2 r_{0}, a=r_{1}+2 r_{0}, a=r_{1}+2 r_{2}$, or $a=r_{-1}+r_{i}+r_{j}$ for $i, j \neq-1,0,1$ and $\boldsymbol{r}_{i} \cdot \boldsymbol{r}_{j}=0$, or $\boldsymbol{a}=\boldsymbol{r}_{1}+\boldsymbol{r}_{i}+\boldsymbol{r}_{j}$ for $i, j \neq-1,0,1,2$ and $\boldsymbol{r}_{i} \cdot \boldsymbol{r}_{j}=0$

$\Rightarrow\left[25 \times(-1)^{4}+3 \times(-1)^{3}\right] \Delta\left(\Lambda_{0}\right)=22 ;$

(c) $\boldsymbol{A} \cdot \boldsymbol{a}=-2$ :

(i) $a^{2}=4$, i.e. $a=r_{-1}+r_{1} \Longrightarrow(-1)^{3} \Delta\left(\Lambda_{0}\right)=-1$.

In total, this gives $-982+1968-1166+126+41+107-117+22-1=-2$ contradicting the conjecture of [1]! We have to conclude that $\boldsymbol{\Lambda}_{1}+\boldsymbol{\delta}$ is an imaginary simple root of multiplicity 2 .

Let $\boldsymbol{A}=\boldsymbol{\Lambda}_{8}$.

(a) $\boldsymbol{A} \cdot a=0$ :

(i) $a=0 \Longrightarrow-\Delta\left(\boldsymbol{A}_{8}\right)=-981$;

(ii) $\boldsymbol{a}^{2}=2$, i.e. $\boldsymbol{a}=\boldsymbol{r}_{i}$ for $i \neq 8 \Longrightarrow 9 \times(-1)^{2} \Delta\left(\boldsymbol{A}_{7}+\boldsymbol{\delta}\right)=2214$,

since $\boldsymbol{A}-\boldsymbol{a} \in \mathfrak{W}\left(\boldsymbol{\Lambda}_{7}+\boldsymbol{\delta}\right)$;

(iii) $\boldsymbol{a}^{2}=4$, i.e. $\boldsymbol{a}=\boldsymbol{r}_{i}+\boldsymbol{r}_{j}$ for $i, j \neq 8$ and $\boldsymbol{r}_{i} \cdot \boldsymbol{r}_{j}=0 \Rightarrow 28 \times(-1)^{3} \Delta\left(\boldsymbol{\Lambda}_{1}\right)=$ -1484 ,

since $\boldsymbol{A}-\boldsymbol{a} \in \mathfrak{W}\left(\boldsymbol{A}_{1}\right)$;

(iv) $\boldsymbol{a}^{2}=6$, i.e. $\boldsymbol{a}=\boldsymbol{r}_{i}+\boldsymbol{r}_{j}+\boldsymbol{r}_{k}$ for $i, j, k \neq 8$ and $\boldsymbol{r}_{i} \cdot \boldsymbol{r}_{j}=\boldsymbol{r}_{j} \cdot \boldsymbol{r}_{k}=\boldsymbol{r}_{k} \cdot \boldsymbol{r}_{i}=0$, or $\boldsymbol{a}=2 \boldsymbol{r}_{i}+\boldsymbol{r}_{j}$ for $i, j \neq 8$ and $\boldsymbol{r}_{i} \cdot \boldsymbol{r}_{j}=-1 \Longrightarrow\left[35 \times(-1)^{4}+16 \times(-1)^{3}\right] \Delta\left(\boldsymbol{A}_{7}\right)=$ 171;

(v) $\boldsymbol{a}^{2}=8$, i.e. $\boldsymbol{a}=\boldsymbol{r}_{i}+\boldsymbol{r}_{j}+\boldsymbol{r}_{k}+\boldsymbol{r}_{l}$ for $i, j, k, l \neq 8$ and $\boldsymbol{r}_{i} \cdot \boldsymbol{r}_{j}=\ldots=\boldsymbol{r}_{l} \cdot \boldsymbol{r}_{i}=0$, or $\boldsymbol{a}=2 \boldsymbol{r}_{i}+\boldsymbol{r}_{j}+\boldsymbol{r}_{k}$ for $i, j, k \neq 8$ and $\boldsymbol{r}_{i} \cdot \boldsymbol{r}_{j}=-1, \boldsymbol{r}_{i} \cdot \boldsymbol{r}_{k}=\boldsymbol{r}_{j} \cdot \boldsymbol{r}_{k}=0$, or

\footnotetext{
${ }^{6}$ Note that this is the first example where we have to take into account the product over the positive roots appearing on the l.h.s. of (13).
} 


$$
\begin{aligned}
& a=2\left(\boldsymbol{r}_{i}+\boldsymbol{r}_{j}\right) \text { for } i, j \neq 8 \text { and } \boldsymbol{r}_{i} \cdot \boldsymbol{r}_{j}=-1 \Rightarrow\left[15 \times(-1)^{5}+84 \times(-1)^{4}+\right. \\
& \left.8 \times(-1)^{4}\right] \Delta\left(\boldsymbol{\Lambda}_{0}\right)=77
\end{aligned}
$$

(b) $\boldsymbol{A} \cdot \boldsymbol{a}=-1$ :

(i) $a^{2}=2$, i.e. $a=r_{8} \Longrightarrow(-1)^{2} \Delta\left(\Lambda_{1}\right)=53$,

since $\boldsymbol{A}-\boldsymbol{r}_{8} \in \mathfrak{W}\left(\boldsymbol{\Lambda}_{1}\right)$;

(ii) $a^{2}=4$, i.e. $a=r_{8}+r_{i}$ for $i \neq 5,8 \Rightarrow 8 \times(-1)^{3} \Delta\left(\Lambda_{7}\right)=-72$;

(iii) $a^{2}=6$, i.e. $a=r_{8}+2 r_{5}$ or $a=r_{8}+r_{i}+r_{j}$ for $i, j \neq 5,8$ and $r_{i} \cdot r_{j}=0$ $\Longrightarrow\left[22 \times(-1)^{4}+1 \times(-1)^{3}\right] \Delta\left(\boldsymbol{A}_{0}\right)=21$.

In total, this gives $-981+2214-1484+171+77+53-72+21=-1$ again contradicting the conjecture. We conclude that $\boldsymbol{\Lambda}_{8}$ is an imaginary simple root of multiplicity 1 .

\section{Simple roots and simple multiplicities for $\Lambda^{2} \geqslant-24$}

The above calculations can now be carried much further with the help of a computer, and in this section we present the results that we have obtained down to norms $\boldsymbol{A}^{2}=$ -24 . Before giving these results, we would, however, like to stress once more some of the extra complications that arise as the root norms become more negative. As is already evident from the example $\boldsymbol{\Lambda}=\boldsymbol{\Lambda}_{1}+\boldsymbol{\delta}$ of the last section, we must now deal with the product over all positive roots appearing on the 1.h.s. of (13). More specifically, for a given $\operatorname{root} \boldsymbol{\Lambda}$ we will have to take into account all decompositions of $\boldsymbol{v}=\boldsymbol{A}-\boldsymbol{a}$ into sums of positive roots. To find them, we make use of the following strategy. Since only positive roots with non-vanishing $\Delta$ contribute, we can disregard all real and lightlike imaginary roots. Moreover, without loss of generality we can rotate $v$ into the fundamental Weyl chamber and look for decompositions $v=\sum_{j} v_{j}$ there; it is important here that the summands $v_{j}$ need not belong to $\mathcal{C}$ separately. Starting from any such decomposition, the action of the little Weyl group $\mathcal{W}(\boldsymbol{A})$ (i.e., the stability subgroup leaving $\boldsymbol{A}$ fixed) yields further decompositions. Hence we have to take into account only those decompositions where at least one of the elements $v_{j}$ is a lowest weight vector of $\mathcal{W}(\boldsymbol{A})$. In the cases we have investigated we could restrict the search even further because only decompositions into two components can occur. In general, however, decompositions into an arbitrary number of positive roots will have to be considered. For roots which are multiples of other roots we also have another contribution, coming from higher terms in the expansion of $\left(1-\mathrm{e}^{v}\right)^{\Delta(v)}$.

Since in long computer calculations ${ }^{7}$ one can never exclude all possible sources of errors, we emphasize once more that the positivity of the final result constitutes an important consistency check, especially with descending norms as the numbers involved in the sum become very large. Our results are collected in Table 1.

For the convenience of the reader and to provide a "bird's eye's" view on the results obtained so far, we have displayed them once more in the table below. This table

\footnotetext{
${ }^{7}$ For instance, the determination of $\mu\left(\boldsymbol{A}_{3}\right)=2$ took one hour of CPU time.
} 
Table 1

Simple multiplicities of imaginary simple roots with $\Lambda^{2} \leqslant-2$

\begin{tabular}{|c|c|c|c|c|c|}
\hline$A$ & $\ell(\boldsymbol{A})$ & $\operatorname{ht}(\boldsymbol{A})$ & $A^{2}$ & $\operatorname{dim} \mathrm{g}_{\mu_{y, 1}}{ }^{(\boldsymbol{A})}$ & $\mu(\boldsymbol{A})$ \\
\hline $\boldsymbol{A}_{0}$ & 1 & 61 & -2 & 45 & 1 \\
\hline$A_{7}$ & 2 & 76 & -4 & 201 & 0 \\
\hline $\boldsymbol{\delta}+\boldsymbol{A}_{0}$ & 1 & 91 & -4 & 201 & 1 \\
\hline$A_{1}$ & 2 & 93 & -6 & 780 & 0 \\
\hline$\delta+\boldsymbol{A}_{7}$ & 2 & 106 & -8 & 2718 & 0 \\
\hline $\boldsymbol{A}_{8}$ & 3 & 115 & -10 & 8730 & 1 \\
\hline $2 \boldsymbol{\delta}+\boldsymbol{A}_{0}$ & 1 & 121 & -6 & 780 & 2 \\
\hline $2 \boldsymbol{A}_{0}$ & 2 & 122 & -8 & 2718 & 0 \\
\hline$\delta+A_{1}$ & 2 & 123 & -10 & 8730 & 2 \\
\hline $\boldsymbol{A}_{2}$ & 3 & 126 & -12 & 26226 & 0 \\
\hline $2 \delta+\Lambda_{7}$ & 2 & 136 & -12 & 26226 & 1 \\
\hline $\boldsymbol{\Lambda}_{0}+\boldsymbol{\Lambda}_{7}$ & 3 & 137 & -14 & 74556 & 3 \\
\hline$\delta+\boldsymbol{A}_{8}$ & 3 & 145 & -16 & 202180 & 3 \\
\hline $3 \boldsymbol{\delta}+\boldsymbol{A}_{0}$ & 1 & 151 & -8 & 2718 & 2 \\
\hline$\delta+2 A_{0}$ & 2 & 152 & -12 & 26226 & 2 \\
\hline $2 \boldsymbol{A}_{7}$ & 4 & 152 & -16 & 202180 & 0 \\
\hline $2 \delta+A_{1}$ & 2 & 153 & -14 & 74556 & 3 \\
\hline $\boldsymbol{A}_{6}$ & 4 & 153 & -18 & 526397 & 3 \\
\hline $\boldsymbol{A}_{0}+\boldsymbol{A}_{\mathbf{l}}$ & 3 & 154 & -16 & 202180 & 4 \\
\hline$\delta+\boldsymbol{A}_{2}$ & 3 & 156 & -18 & 526397 & 6 \\
\hline $\boldsymbol{A}_{3}$ & 4 & 160 & -20 & 1322343 & 2 \\
\hline $3 \boldsymbol{\delta}+\boldsymbol{A}_{7}$ & 2 & 166 & -16 & 202180 & 4 \\
\hline$\delta+\boldsymbol{A}_{0}+\boldsymbol{A}_{7}$ & 3 & 167 & -20 & 1322343 & 14 \\
\hline$A_{1}+A_{7}$ & 4 & 169 & -22 & 3218091 & 14 \\
\hline $2 \delta+\Lambda_{8}$ & 3 & 175 & -22 & 3218091 & 14 \\
\hline $\boldsymbol{A}_{0}+\boldsymbol{\Lambda}_{8}$ & 4 & 176 & -24 & 7612014 & 15 \\
\hline $4 \delta+A_{0}$ & 1 & 181 & -10 & 8730 & 4 \\
\hline $2 \boldsymbol{\delta}+2 \boldsymbol{A}_{0}$ & 2 & 182 & -16 & 202180 & 4 \\
\hline$\delta+2 \Lambda_{7}$ & 4 & 182 & -24 & 7612014 & 9 \\
\hline $3 \delta+A_{1}$ & 2 & 183 & -18 & 526397 & 11 \\
\hline $3 \boldsymbol{A}_{0}$ & 3 & 183 & -18 & 526397 & 7 \\
\hline $\boldsymbol{\delta}+\boldsymbol{A}_{6}$ & 4 & 183 & -26 & 17548920 & \\
\hline $4 \delta+A_{7}$ & 2 & 196 & -20 & 1322343 & 8 \\
\hline $3 \boldsymbol{\delta}+2 \boldsymbol{A}_{0}$ & 2 & 212 & -20 & 1322343 & 13 \\
\hline $4 \delta+\boldsymbol{A}_{1}$ & 2 & 213 & -22 & 3218091 & 25 \\
\hline $5 \delta+A_{7}$ & 2 & 226 & -24 & 5717880 & 19 \\
\hline
\end{tabular}

highlights two facts, namely, (i) that in some cases the simple multiplicities depend only on the norm of the root in question, and (ii) (somewhat to our surprise) that the simple multiplicities at level $\ell=2$ depend also on the "direction" of the root, unlike the corresponding $E_{10}$ multiplicities!

While no clear pattern is discernible in the simple root multiplicities so far, the smallness of the numbers obtained is noteworthy. Especially the zeroes in this table (the corresponding roots thus must not be counted as simple roots) appear to us quite striking in view of the fact that we are unaware of any obvious a priori reason for their existence. Furthermore, we observe that the simple multiplicities do not depend monotonically on the norms for levels $\ell \geqslant 2$, unlike the level-1 simple multiplicities, and 
Table 2

Simple multiplicities of imaginary simple roots for $\mathfrak{g}_{/ I_{9,1}}$

\begin{tabular}{llllllllllllll}
\hline$\ell$ & \multicolumn{10}{c}{$\Lambda^{2}$} \\
\cline { 2 - 12 }$y$ & -2 & -4 & -6 & -8 & -10 & -12 & -14 & -16 & -18 & -20 & -22 & -24 \\
\hline 1 & 1 & 1 & 2 & 2 & 4 & 4 & 7 & 8 & 12 & 14 & 21 & 24 \\
2 & & 0 & 0 & $0^{a}$ & 2 & 1,2 & 3 & $4^{a}$ & 11 & 8,13 & 25 & 19 \\
3 & & & & & 1 & 0 & 3 & 3,4 & 6,7 & 14 & 14 & \\
4 & & & & & & & & 0 & 3 & 2 & 14 & 9,15 \\
\hline
\end{tabular}

accurs twice.

unlike the simple multiplicities of the gnome Lie algebra. Also, for fixed $\boldsymbol{\Lambda}^{2}$, there is a tendency for the simple multiplicities to decrease as a function of the level. Of course, these results might just be a coincidence, and the simple multiplicities, although rather well behaved for low norms and low levels, might explode after a few more steps. On the other hand, the fact that we stay so close to zero makes us wonder if there is not a hidden structure "just around the corner."

The smallness of the simple root multiplicities means that that $E_{10}$ is a rather "big" subalgebra of $\mathfrak{g}_{H_{9,1}}$. This behavior is to be contrasted with that of the gnome Lie algebra $\mathfrak{g}_{H_{1,1}}$, whose maximal Kac Moody subalgebra is the finite algebra $\operatorname{sl}(2, \mathbb{R})$. However, readers should keep in mind that (1) admits an infinite nested sequence of Borcherds algebras "between" $E_{10}$ and $\mathfrak{g}_{l_{9,1}}$ : these are simply obtained obtained by omitting any number of missing $E_{10}$ modules from $\mathfrak{g}_{/ h_{9,1}}$ or, equivalently, the corresponding simple roots from the root system of $\mathfrak{g}_{I_{9,1},}$.

Being confronted with the ineluctable conclusion that $E_{10}$ is much more complicated than either the gnome or the fake monster, the next question is, how should one proceed from here onwards? Recall that the nice structure underlying the root system of the fake monster Lie algebra was discovered by methods very similar to the ones employed here (see remarks in Section 5 of Ref. [3]). By computing the simple multiplicities of roots down to norm -6 , Borcherds realized that the imaginary simple roots are all proportional to $\boldsymbol{\rho}$ with uniform multiplicity 24 (corresponding to the 24 transverse polarizations of a photon in 26 dimensions), where $\boldsymbol{\rho}$ is the lightlike Weyl vector of $I_{25,1}$; and, happily, the pattern thus reveals itself after only very few steps! (Observe that, in fact, for the fake monster, all entries in Table 2 would vanish because $\mathfrak{g}_{I_{25,1}}$ has no simple roots of negative norm.) For $E_{10}$, we are evidently not in such a fortuitous situation, and at this point the only feasible way to make further progress with presently available techniques seems to be to collect even more data about the simple multiplicities. Fortunately, we have seen that our method can be conveniently implemented on a computer.

Assuming a general pattern for the simple multiplicities we would still face the problem of a rigorous proof. For the monster, Borcherds was able to prove that the emerging pattern was, in fact, a general property of the algebra and its root system by establishing a new modular identity. In the case at hand, the question is therefore whether our new denominator formula (13) admits a modular interpretation, too. This question 
is obviously of a more general interest, as similar modified denominator formulas are expected to exist for other algebras of this type. After a suitable specialization, these formulas would give rise to new modular identities involving all levels simultaneously. In making these speculations, we are encouraged by the fact that there do exist examples of automorphic forms which give rise to Borcherds algebras with $E_{10}$ as maximal KacMoody subalgebra (see Example 1 in Section 16 of Refs. [6,10]).

\section{Acknowledgements}

We are indebted to R. Borcherds for sharing with us his (unpublished) expertise on reflection groups and the Peterson formula, and to $J$. Fuchs for explaining to us his Weyl orbit method. H.N. would like to thank the Newton Institute in Cambridge, where part of this work was carried out, for hospitality and support. O.B. is grateful to the Albert-Einstein-Institut for hospitality during a visit there.

\section{Appendix A. $E_{10}$ Multiplicities for $\Lambda \in \mathcal{C}$ with $h t(\Lambda) \leqslant 231$}

In this appendix we collect the multiplicities of all roots of $E_{10}$ with height $\leqslant 231$. This includes the multiplicities of all fundamental weights of $E_{10}$.

The calculation of these multiplicities starts from Peterson's formula, which in principle allows the recursive computation of the multiplicity of any root. For algebras of high rank such as $E_{10}$, however, this procedure soon takes up too much time due to the large number of roots involved. We will use an approach due to Borcherds [7] to simplify the calculations. The idea is to employ the little Weyl (or stability) group of the root in question and to group the roots into orbits of this group and then count these orbits rather than the roots themselves. One has the following identity

$$
(\boldsymbol{\Lambda} \mid \boldsymbol{A}-2 \boldsymbol{\rho}) c_{\boldsymbol{\Lambda}}=\sum_{\substack{\boldsymbol{\beta}^{\prime}, \boldsymbol{\beta}^{\prime \prime} \in Q_{+}^{\prime} \\ \boldsymbol{A}=\boldsymbol{\beta}^{\prime}+\boldsymbol{\beta}^{\prime \prime}}}\left(\boldsymbol{\beta}^{\prime} \mid \boldsymbol{\beta}^{\prime \prime}\right) c_{\boldsymbol{\beta}^{\prime}} c_{\boldsymbol{\beta}^{\prime \prime}} \stackrel{!}{=} \sum_{\boldsymbol{v} \in Q_{+}}(\boldsymbol{v} \mid \boldsymbol{\Lambda}-\boldsymbol{v}) c_{\boldsymbol{v}} c_{\boldsymbol{A}-\boldsymbol{v}}|\mathcal{W}(\boldsymbol{v})|
$$

The second sum is over all real roots and all lowest weight vectors $v$ in $Q_{+}$with respect to the little Weyl group $\mathcal{W}(\boldsymbol{\Lambda})$ such that $\boldsymbol{\Lambda}-\boldsymbol{v}$ is also a positive root. One also has to be careful not to count the same orbit twice. $|\mathcal{W}(v)|$ denotes the size of the orbit of this lowest weight vector under $\mathcal{W}(\boldsymbol{\Lambda})$ and we have

$$
c_{\boldsymbol{\beta}}=\sum_{k \geqslant 1} \frac{1}{k} \operatorname{mult}\left(\frac{\boldsymbol{\beta}}{k}\right) .
$$

To give an example for this procedure we consider the two simplest cases: $\boldsymbol{A}=\boldsymbol{\Lambda}_{-1}=$ $\boldsymbol{\delta}$ and $\boldsymbol{\Lambda}=\boldsymbol{\Lambda}_{0}$.

(1) $\boldsymbol{\Lambda}=\boldsymbol{\delta} \Rightarrow(\boldsymbol{\Lambda} \mid \boldsymbol{\Lambda}-2 \boldsymbol{\rho})=-60$

(a) $\delta=\boldsymbol{r}_{0}+\boldsymbol{\theta} \Rightarrow(\boldsymbol{\theta} \mid \boldsymbol{A}-\boldsymbol{\theta}) c_{\boldsymbol{\theta}} c_{\boldsymbol{A}-\boldsymbol{\theta}}|\mathcal{W}(\boldsymbol{\theta})|=-240$ 
Table A.1

$E_{10}$ root multiplicities

\begin{tabular}{|c|c|c|c|c|c|c|}
\hline $\boldsymbol{A}$ & $\boldsymbol{A}$ & $\ell(\boldsymbol{A})$ & $\operatorname{ht}(\boldsymbol{A})$ & $\boldsymbol{A}^{2}$ & mult $(A$ & $\Delta(\boldsymbol{A})$ \\
\hline$\delta$ & {$[0,1,2,3,4,5,6,4,2,3]$} & 0 & 30 & 0 & 8 & 0 \\
\hline $2 \delta$ & {$[0,2,4,6,8,10,12,8,4,6]$} & 0 & 60 & 0 & 8 & 0 \\
\hline $\boldsymbol{A}_{0}$ & {$[1,2,4,6,8,10,12,8,4,6]$} & 1 & 61 & -2 & 44 & 1 \\
\hline$A_{7}$ & {$[2,4,6,8,10,12,14,9,4,7]$} & 2 & 76 & -4 & 192 & 9 \\
\hline $3 \delta$ & {$[0,3,6,9,12,15,18,12,6,9]$} & 0 & 90 & 0 & 8 & 0 \\
\hline $\boldsymbol{\delta}+\boldsymbol{\Lambda}_{0}$ & {$[1,3,6,9,12,15,18,12,6,9]$} & 1 & 91 & -4 & 192 & 9 \\
\hline $\boldsymbol{A}_{1}$ & {$[2,4,6,9,12,15,18,12,6,9]$} & 2 & 93 & -6 & 727 & 53 \\
\hline $\boldsymbol{\delta}+\boldsymbol{A}_{7}$ & {$[2,5,8,11,14,17,20,13,6,10]$} & 2 & 106 & -8 & 2472 & 246 \\
\hline $\boldsymbol{A}_{8}$ & {$[3,6,9,12,15,18,21,14,7,10]$} & 3 & 115 & -10 & 7749 & 981 \\
\hline $4 \delta$ & {$[0,4,8,12,16,20,24,16,8,12]$} & 0 & 120 & 0 & 8 & 0 \\
\hline $2 \delta+\boldsymbol{A}_{0}$ & {$[1,4,8,12,16,20,24,16,8,12]$} & 1 & 121 & -6 & 726 & 54 \\
\hline $2 \Lambda_{0}$ & {$[2,4,8,12,16,20,24,16,8,12\}$} & 2 & 122 & -8 & 2472 & 246 \\
\hline$\delta+\boldsymbol{A}_{1}$ & {$[2,5,8,12,16,20,24,16,8,12]$} & 2 & 123 & -10 & 7747 & 983 \\
\hline $\boldsymbol{\Lambda}_{2}$ & $\{3,6,9,12,16,20,24,16,8,12\}$ & 3 & 126 & -12 & 22725 & 3501 \\
\hline $2 \delta+A_{7}$ & $\{2,6,10,14,18,22,26,17,8,13\}$ & 2 & 136 & -12 & 22712 & 3514 \\
\hline $\boldsymbol{A}_{0}+\boldsymbol{A}_{7}$ & $\{3,6,10,14,18,22,26,17,8,13\}$ & 3 & 137 & -14 & 63085 & 11471 \\
\hline $\boldsymbol{\delta}+\boldsymbol{A}_{8}$ & {$[3,7,11,15,19,23,27,18,9,13]$} & 3 & 145 & -16 & 167116 & 35064 \\
\hline $5 \delta$ & {$[0,5,10,15,20,25,30,20,10,15]$} & 0 & 150 & 0 & 8 & 0 \\
\hline $3 \delta+\Lambda_{0}$ & {$[1,5,10,15,20,25,30,20,10,15]$} & 1 & 151 & -8 & 2464 & 254 \\
\hline$\delta+2 A_{0}$ & {$[2,5,10,15,20,25,30,20,10,15]$} & 2 & 152 & -12 & 22712 & 3514 \\
\hline $2 \boldsymbol{A}_{7}$ & {$[4,8,12,16,20,24,28,18,8,14]$} & 4 & 152 & -16 & 167133 & 35047 \\
\hline $2 \delta+\boldsymbol{A}_{\mathrm{l}}$ & {$[2,6,10,15,20,25,30,20,10,15]$} & 2 & 153 & -14 & 63020 & 11536 \\
\hline $\mathbf{A}_{6}$ & {$[4,8,12,16,20,24,28,18,9,14\rceil$} & 4 & 153 & -18 & 425227 & 101170 \\
\hline$\Lambda_{0}+\Lambda_{1}$ & {$[3,6,10,15,20,25,30,20,10,15]$} & 3 & 154 & -16 & 167099 & 35081 \\
\hline$\delta+\boldsymbol{A}_{2}$ & {$[3,7,11,15,20,25,30,20,10,15]$} & 3 & 156 & -18 & 425156 & 101241 \\
\hline $\boldsymbol{A}_{3}$ & {$[4,8,12,16,20,25,30,20,10,15]$} & 4 & 160 & -20 & 1044218 & 278125 \\
\hline $3 \boldsymbol{\delta}+\boldsymbol{A}_{7}$ & {$[2,7,12,17,22,27,32,21,10,16]$} & 2 & 166 & -16 & 166840 & 35340 \\
\hline$\delta+\boldsymbol{A}_{0}+\boldsymbol{A}_{7}$ & {$[3,7,12,17,22,27,32,21,10,16]$} & 3 & 167 & -20 & 1043926 & 278417 \\
\hline $\boldsymbol{A}_{1}+\boldsymbol{\Lambda}_{7}$ & {$[4,8,12,17,22,27,32,21,10,16]$} & 4 & 169 & -22 & 2485020 & 733071 \\
\hline $2 \delta+A_{8}$ & {$[3,8,13,18,23,28,33,22,11,16]$} & 3 & 175 & -22 & 2483970 & 734121 \\
\hline $\boldsymbol{A}_{0}+\boldsymbol{A}_{8}$ & {$[4,8,13,18,23,28,33,22,11,16]$} & 4 & 176 & -24 & 5749818 & 1862196 \\
\hline $6 \delta$ & {$[0,6,12,18,24,30,36,24,12,18]$} & 0 & 180 & 0 & 8 & 0 \\
\hline $4 \delta+\Lambda_{0}$ & {$[1,6,12,18,24,30,36,24,12,18]$} & 1 & 181 & -10 & 7704 & 1026 \\
\hline $2 \delta+2 \Lambda_{0}$ & {$[2,6,12,18,24,30,36,24,12,18]$} & 2 & 182 & -16 & 166840 & 35340 \\
\hline$\delta+2 \boldsymbol{A}_{7}$ & {$[4,9,14,19,24,29,34,22,10,17]$} & 4 & 182 & -24 & 5750072 & 1861942 \\
\hline $3 \delta+\Lambda_{1}$ & {$[2,7,12,18,24,30,36,24,12,18]$} & 2 & 183 & -18 & 424161 & 102236 \\
\hline $3 \Lambda_{0}$ & {$[3,6,12,18,24,30,36,24,12,18]$} & 3 & 183 & -18 & 425058 & 101339 \\
\hline$\delta+\boldsymbol{A}_{6}$ & {$[4,9,14,19,24,29,34,22,11,17]$} & 4 & 183 & -26 & 12971009 & 4577911 \\
\hline$\delta+\boldsymbol{A}_{0}+\boldsymbol{A}_{1}$ & {$[3,7,12,18,24,30,36,24,12,18]$} & 3 & 184 & -22 & 248387 I & 734220 \\
\hline $2 \delta+\Lambda_{2}$ & $|3,8,13,18,24,30,36,24,12,18|$ & 3 & 186 & -24 & 5746226 & 1865788 \\
\hline $2 \boldsymbol{A}_{1}$ & $|4,8,12,18,24,30,36,24,12,18|$ & 4 & 186 & -24 & 5749565 & 1862449 \\
\hline $\boldsymbol{A}_{0}+\boldsymbol{A}_{2}$ & {$[4,8,13,18,24,30,36,24,12,18]$} & 4 & 187 & -26 & 12970045 & 4578875 \\
\hline$\delta+\boldsymbol{A}_{3}$ & {$[4,9,14,19,24,30,36,24,12,18]$} & 4 & 190 & -28 & 28592513 & 10931086 \\
\hline $\boldsymbol{A}_{7}+\boldsymbol{A}_{8}$ & {$[5,10,15,20,25,30,35,23,11,17]$} & 5 & 191 & -28 & 28595548 & 10928051 \\
\hline $\mathbf{A}_{4}$ & {$[5,10,15,20,25,30,36,24,12,18]$} & 5 & 195 & -30 & 61721165 & 25411831 \\
\hline $4 \boldsymbol{\delta}+\boldsymbol{\Lambda}_{7}$ & {$[2,8,14,20,26,32,38,25,12,19]$} & 2 & 196 & -20 & 1040664 & 281679 \\
\hline $2 \boldsymbol{\delta}+\boldsymbol{\Lambda}_{0}+\boldsymbol{\Lambda}_{7}$ & {$[3,8,14,20,26,32,38,25,12,19]$} & 3 & 197 & -26 & 12959290 & 4589630 \\
\hline
\end{tabular}


Table A.1 - continued

\begin{tabular}{|c|c|c|c|c|c|c|}
\hline $\boldsymbol{A}$ & $\boldsymbol{A}$ & $\ell(\boldsymbol{A})$ & ht $(\boldsymbol{A})$ & $A^{2}$ & $\operatorname{mult}(\boldsymbol{A})$ & $\Delta(\boldsymbol{A})$ \\
\hline $2 \boldsymbol{A}_{0}+\boldsymbol{A}_{7}$ & {$[4,8,14,20,26,32,38,25,12,19]$} & 4 & 198 & -28 & 28589025 & 10934574 \\
\hline$\delta+\Lambda_{1}+\Lambda_{7}$ & {$[4,9,14,20,26,32,38,25,12,19]$} & 4 & 199 & -30 & 61711591 & 25421405 \\
\hline $\mathbf{A}_{2}+\mathbf{A}_{7}$ & {$[5,10,15,20,26,32,38,25,12,19]$} & 5 & 202 & -32 & 130661924 & 57690454 \\
\hline $3 \delta+\Lambda_{8}$ & {$[3,9,15,21,27,33,39,26,13,19]$} & 3 & 205 & -28 & 28559052 & 10964547 \\
\hline$\delta+\boldsymbol{\Lambda}_{0}+\boldsymbol{A}_{8}$ & {$[4,9,15,21,27,33,39,26,13,19]$} & 4 & 206 & -32 & 130632964 & 57719414 \\
\hline $\mathbf{A}_{1}+\mathbf{A}_{8}$ & {$[5,10,15,21,27,33,39,26,13,19]$} & 5 & 208 & -34 & 271695444 & 128129588 \\
\hline $7 \delta$ & $10,7,14,21,28,35,42,28,14,21]$ & 0 & 210 & 0 & 8 & 0 \\
\hline $5 \delta+A_{0}$ & {$[1,7,14,21,28,35,42,28,14,21]$} & $\mathbf{l}$ & 211 & -12 & 22528 & 3698 \\
\hline $3 \delta+2 \Lambda_{0}$ & {$[2,7,14,21,28,35,42,28,14,21]$} & 2 & 212 & -20 & 1040664 & 281679 \\
\hline $2 \delta+2 A_{7}$ & {$[4,10,16,22,28,34,40,26,12,20]$} & 4 & 212 & -32 & 130635596 & 57716782 \\
\hline $4 \delta+\boldsymbol{A}_{1}$ & {$[2,8,14,21,28,35,42,28,14,21]$} & 2 & 213 & -22 & 2474026 & 744065 \\
\hline$\delta+3 \boldsymbol{\Lambda}_{0}$ & {$[3,7,14,21,28,35,42,28,14,21]$} & 3 & 213 & -24 & 5745720 & 1866294 \\
\hline $\boldsymbol{A}_{0}+2 \boldsymbol{A}_{7}$ & {$[5,10,16,22,28,34,40,26,12,20]$} & 5 & 213 & -34 & 271702532 & 128122500 \\
\hline $2 \delta+\boldsymbol{A}_{6}$ & {$[4,10,16,22,28,34,40,26,13,20]$} & 4 & 213 & -34 & 271618575 & 128206457 \\
\hline $2 \delta+A_{0}+A_{1}$ & $\{3,8,14,21,28,35,42,28,14,21\}$ & 3 & 214 & -28 & 28558597 & 10965002 \\
\hline $\boldsymbol{\Lambda}_{11}+\boldsymbol{A}_{6}$ & {$[5,10,16,22,28,34,40,26,13,20\}$} & 5 & 214 & -36 & 555652661 & 278885204 \\
\hline $2 \boldsymbol{A}_{0}+\boldsymbol{A}_{1}$ & {$[4,8,14,21,28,35,42,28,14,21]$} & 4 & 215 & -30 & 61699285 & 25433711 \\
\hline $3 \boldsymbol{\delta}+\boldsymbol{A}_{2}$ & {$[3,9,15,21,28,35,42,28,14,21]$} & 3 & 216 & -30 & 61620301 & 25512695 \\
\hline$\delta+2 \Lambda_{1}$ & $\{4,9,14,21,28,35,42,28,14,21\}$ & 4 & 216 & -32 & 130630342 & 57722036 \\
\hline$\delta+\boldsymbol{\Lambda}_{0}+\boldsymbol{\Lambda}_{2}$ & {$[4,9,15,21,28,35,42,28,14,21]$} & 4 & 217 & -34 & 271609694 & 128215338 \\
\hline $\boldsymbol{A}_{1}+\boldsymbol{A}_{2}$ & $|5,10,15,21,28,35,42,28,14,21|$ & 5 & 219 & -36 & 555631102 & 278906763 \\
\hline $2 \delta+\Lambda_{3}$ & {$[4,10,16,22,28,35,42,28,14,21]$} & 4 & 220 & -36 & 555434128 & 279103737 \\
\hline$\delta+\boldsymbol{\Lambda}_{7}+\boldsymbol{\Lambda}_{8}$ & $\mid 5,11,17,23,29,35,41,27,13,20]$ & 5 & 221 & -38 & 1118955631 & 595793867 \\
\hline $\mathbf{A}_{1}+\mathbf{A}_{3}$ & {$[5,10,16,22,28,35,42,28,14,21]$} & 5 & 221 & -38 & 1118894437 & 595855061 \\
\hline$\delta+\Lambda_{4}$ & {$[5,11,17,23,29,35,42,28,14,21]$} & 5 & 225 & -40 & 2220872914 & 1251118823 \\
\hline $5 \delta+A_{7}$ & {$[2,9,16,23,30,37,44,29,14,22]$} & 2 & 226 & -24 & 5717880 & 1894134 \\
\hline $3 \boldsymbol{\delta}+\boldsymbol{A}_{0}+\boldsymbol{A}_{7}$ & {$[3,9,16,23,30,37,44,29,14,22]$} & 3 & 227 & -32 & 130395100 & 57957278 \\
\hline $\boldsymbol{\delta}+2 \boldsymbol{A}_{0}+\boldsymbol{A}_{7}$ & {$[4,9,16,23,30,37,44,29,14,22]$} & 4 & 228 & -36 & 555404364 & 279133501 \\
\hline $3 \boldsymbol{A}_{7}$ & {$[6,12,18,24,30,36,42,27,12,21]$} & 6 & 228 & -36 & 555695680 & 278842185 \\
\hline $2 \boldsymbol{\delta}+\boldsymbol{A}_{1}+\boldsymbol{A}_{7}$ & {$[4,10,16,23,30,37,44,29,14,22]$} & 4 & 229 & -38 & 1118347860 & 596401638 \\
\hline $\boldsymbol{A}_{6}+\boldsymbol{A}_{7}$ & $|6,12,18,24,30,36,42,27,13,21|$ & 6 & 229 & -40 & 2221039540 & 1250952197 \\
\hline $\boldsymbol{A}_{0}+\boldsymbol{A}_{1}+\boldsymbol{A}_{7}$ & {$[5,10,16,23,30,37,44,29,14,22]$} & 5 & 230 & -40 & 2220699951 & 1251291786 \\
\hline $2 \boldsymbol{A}_{8}$ & {$[6,12,18,24,30,36,42,28,14,20]$} & 6 & 230 & -40 & 2221026189 & 1250965548 \\
\hline $\boldsymbol{A}_{5}$ & $|6,12,18,24,30,36,42,28,14,21|$ & 6 & 231 & -42 & $4348985101 \quad 2$ & 2584919075 \\
\hline
\end{tabular}

Taking an extra factor of 2 due to the symmetry of the sum into account we recover the well-known result mult $(\delta)=8$.

(2) $\boldsymbol{\Lambda}=\boldsymbol{\Lambda}_{0} \Rightarrow(\boldsymbol{\Lambda} \mid \boldsymbol{\Lambda}-2 \boldsymbol{\rho})=-124$
(a) $\boldsymbol{\Lambda}_{0}=\boldsymbol{\delta}+\left(\boldsymbol{\Lambda}_{0}-\delta\right) \Rightarrow(\boldsymbol{\delta} \mid \boldsymbol{\Lambda}-\boldsymbol{\delta}) c_{\delta} c_{\Lambda-\delta}|\mathcal{W}(\boldsymbol{\delta})|=-64$
(b) $\boldsymbol{\Lambda}_{0}=\boldsymbol{r}_{-1}+\left(\boldsymbol{\Lambda}_{0}-\boldsymbol{r}_{-1}\right) \Rightarrow\left(\boldsymbol{r}_{-1} \mid \boldsymbol{\Lambda}-\boldsymbol{r}_{-1}\right) c_{\boldsymbol{r}_{-1}} c_{\boldsymbol{A}-\boldsymbol{r}_{-1}}\left|\mathcal{W}\left(\boldsymbol{r}_{-1}\right)\right|=-24$
(c) $\boldsymbol{\Lambda}_{0}=\boldsymbol{r}_{0}+\left(\boldsymbol{A}_{0}-\boldsymbol{r}_{0}\right) \Rightarrow\left(\boldsymbol{r}_{0} \mid \boldsymbol{A}-\boldsymbol{r}_{0}\right) c_{r_{0}} c_{\boldsymbol{A}-\boldsymbol{r}_{0}}\left|\mathcal{W}\left(\boldsymbol{r}_{0}\right)\right|=-1920$
(d) $\boldsymbol{A}_{0}=\boldsymbol{r}_{1}+\left(\boldsymbol{A}_{0}-\boldsymbol{r}_{1}\right) \Rightarrow\left(\boldsymbol{r}_{1} \mid \boldsymbol{\Lambda}-\boldsymbol{r}_{1}\right) c_{r_{1}} c_{\boldsymbol{A}-\boldsymbol{r}_{1}}\left|\mathcal{W}\left(\boldsymbol{r}_{1}\right)\right|=-720$

Taking an extra factor of 2 due to the symmetry of the sum into account we find the expected result mult $\left(\boldsymbol{\Lambda}_{0}\right)=44$.

For a given $\boldsymbol{v}$ the size of the Weyl orbit is easily calculated as follows: For $\boldsymbol{A} \neq \boldsymbol{\delta}$ the little Weyl group is a finite group with known order. We can also assume that $\boldsymbol{v}$ is a lowest weight vector for this group. The subgroup fixing it is then the Weyl group 
whose simple roots are those orthogonal to the vector $v$. The order of this subgroup is calculated by looking it up, determining the simple factors from the Dynkin diagram. The size of the orbit is then given as the quotient of these two orders.

The remaining problem is to find all lowest weight vectors for $\mathcal{W}(\boldsymbol{\Lambda})$ in $Q_{+}$. These are given by all positive roots of the form

$$
v=\sum_{i=-1}^{8} n_{i} \boldsymbol{\Lambda}_{i}
$$

where only the coefficients $n_{i}$ corresponding to simple roots $\boldsymbol{r}_{i}$ orthogonal to $\boldsymbol{A}$ have to be positive. Due to the symmetry of the sum one can restrict the search to vectors with heights ht $(v) \leqslant[h t(\Lambda) / 2]$.

For roots of low height this formula can be evaluated by hand but this becomes impractical very quickly due to the large number of orbits. For $\boldsymbol{A}=\boldsymbol{\Lambda}_{0}+\boldsymbol{\Lambda}_{1}+\boldsymbol{\Lambda}_{7}$ e.g. there are 635 contributing Weyl orbits. Hence most of the multiplicities were calculated with a computer using the symbolic algebra system Maple V. One consistency check here is that, despite the occurrence of fractional numbers at intermediate stages of the calculation, the final result must be an integer.

Our results for the $E_{10}$ multiplicities and the values of $\Delta$ (cf. (11)) are collected in Table A.1, where we have labeled the root $\Lambda=\sum_{j=-1}^{8} n_{j} \boldsymbol{\Lambda}_{j}$ by the symbol $\left[n_{-1}, n_{0}, n_{1}, \ldots, n_{8}\right]$.

\section{References}

[1] O. Bärwald, R.W. Gebert, M. Günaydin and H. Nicolai, Missing Modules, the Gnome Lie Algebra, and $E_{10}$, preprint KCL-MTH-97-22, IASSNS-HEP-97/20, PSU-TH-178, AEI-029, hep-th/9703084, to appear in Commun. Math. Phys.

[2] M. Bauer and D. Bernard, On root multiplicities of some hyperbolic Kac-Moody algebras, preprint SPhT-96-145, hep-th/9612210 (1996).

[3] R.E. Borcherds, J. Algebra 115 (1988) 501.

[4] R.E. Borcherds, Adv. in Math. 83 (1990) 30.

[5] R.E. Borcherds, Invent. Math. 109 (1992) 405.

[6] R.E. Borcherds, Invent. Math. 120 (1995) 161.

17] R.E. Borcherds, private communication.

[8] J. Fuchs, private communication.

19] R.W. Gebert, H. Nicolai and P.C. West, Int. J. Mod. Phys. A 11 ( 1996 ) 429.

[10] J.A. Harvey and G. Moore, Nucl. Phys. B 463 ( 1996) 315.

[11] E. Jurisich, Generalized Kac-Moody Lie Algebras, Free Lie Algebras and the Structure of the Monster Lie Algebra, to appear in J. Pure Appl. Algebra 122 (1997).

[12] V.G. Kac, Infinite Dimensional Lie Algebras, 3rd ed. (Cambridge University Press, Cambridge, 1990).

[13] V.G. Kac, R.V. Moody and M. Wakimoto, On $E_{10}$, in Differential Geometrical Methods in Theoretical Physics, Proc. NATO Advanced Research Workshop, 16th Int. Conf., Como, ed. K. Bleuler and M. Werner (Kluwer, Holland, 1988) pp. 109-128.

[14] S. Kass, R.V. Moody, J. Patera and R. Slansky, Affine Lie Algebras, Weight Multiplicities, and Branching Rules, Vol. 1\&2 (University of California Press, Berkeley, 1990). 